\title{
STUDY ON THE LONGITUDINAL IMPEDANCE OF BPM FOR KEKB AND SUPER KEKB
}

\author{
K. Shibata ${ }^{\#}$, M. Tobiyama, M. Tejima, Y. Suetsugu, H. Fukuma, S. Hiramatsu \\ KEK, Tsukuba, Japan
}

\section{Abstract}

The longitudinal impedance of KEK B-factory (KEKB) button-type beam position monitors (BPMs) was recalculated by MAFIA in preparation for a future plan to increase the beam current. The diameter and the gap of the button electrode were $12 \mathrm{~mm}$ and $1 \mathrm{~mm}$, respectively. For High Energy Ring (HER), an asymmetric structure was applied to extract the TE110 mode into the coaxial cable. The Q-value and shunt impedance were estimated at 91 and $17 \mathrm{ohm}$ (at $7.6 \mathrm{GHz}$ ), respectively. In this case, the current limit was $2.6 \mathrm{~A}$. Based on the experiences at the KEKB, the new BPM was designed for the Super $\mathrm{KEKB}$, a future high-intensity B-factory at KEK. In order to reduce the impedance the electrode diameter was cut down to $6 \mathrm{~mm}$ from $12 \mathrm{~mm}$. the Q-value and shunt impedance were estimated at 23 and $2 \mathrm{ohm}$ (at $13 \mathrm{GHz}$ ), respectively. The current limit was expected to be about 7 $\mathrm{A}$ in full bucket operation (5120 bunches), and more than $10 \mathrm{kA}$ in 4-bucket spacing operation.

\section{INTRODUCTION}

The beam currents of KEKB have been gradually pushed up in order to get higher luminosity. At present the maximum beam current of Low Energy Ring (LER) is $2000 \mathrm{~mA}$ (1500 bunches) which is lower than the design current (2600 mA ,5120 bunches). On the other hand, the maximum beam current of HER exceeded the design value (1100 mA, 5120 bunches) and reached $1400 \mathrm{~mA}$ (1389 bunches). After this summer shutdown, further increment of the beam currents is planed. In the preparation for the current increment, it is necessary to recalculate the longitudinal impedance of BPMs which may cause the longitudinal multi bunch instability (MBI) and estimate the acceptable beam current. Here we calculated the longitudinal impedance of the BPM for HER by using MAFIA T3 and Eigenmode solvers, and then the current limit due to the longitudinal MBI was estimated.

Looking to the future high-intensity machine, however, it is required to develop a BPM with further lower impedance in order to store much higher beam currents. We also calculated the impedance of BPMs varying the electrode diameter and the gap between the electrode and the wall. Based on this calculation, we made the test models and they will be installed in KEKB during this summer shutdown.

${ }^{{ } k y o . s h i b a t a @ k e k . j p ~}$

\section{SIMULATION}

\section{Impedance and Current Limit}

The Q-value, the shunt impedance and the resonance frequency were estimated by using MAFIA T3 and Eigenmode solvers. The T3 solver consists of two kinds of calculations, namely the wake field and the port transmission calculations [1]. The Q-value estimated by this solver, $Q_{\mathrm{T} 3}$, is given by

$$
1 / Q_{\mathrm{T} 3}=1 / Q_{\mathrm{rad}}+1 / Q_{\text {trans }},
$$

where $Q_{\text {rad }}$ is the Q-value related to the electromagnetic fields which are excited at the BPM and acts back the beam, and $Q_{\text {trans }}$ comes from the electromagnetic waves outgoing from the coaxial cable of the BPM. On the other hand, the Q-value which comes from the energy losses in BPM must be estimated by the Eigenmode solver, since the T3 solver does not take into account the energy losses in materials. The total Q-value is given by

$$
1 / Q_{\text {total }}=1 / Q_{\mathrm{T} 3}+1 / Q_{\mathrm{E}}
$$

where $Q_{\mathrm{E}}$ is the Q-value estimated by Eigenmode solver. Meanwhile, the total shunt impedance, $R_{\text {s_total }}$, is given by

$$
R_{\text {s_total }}=\left(Q_{\text {total }} / Q_{\mathrm{T} 3}\right) R_{\text {s_ } \_33},
$$

where $R_{\mathrm{S}_{-} \mathrm{T} 3}$ is the shunt impedance calculated by T3 solver, because the $R_{\mathrm{s}} / Q$ value depends solely on the geometric structure of BPM.

From the Fourier transform of the wake potential calculated by the T3 solver, we obtain the impedance curve. The values of $Q_{\mathrm{T} 3}$ and $R_{\mathrm{S} \_\mathrm{T} 3}$ are estimated by fitting this curve to

$$
Z^{\prime \prime}(\omega)=\frac{R_{s}}{1+i Q\left(\omega_{R} / \omega-\omega / \omega_{R}\right)},
$$

where $\omega_{\mathrm{R}}$ is the resonance frequency.

The growth rate of the longitudinal MBI for a regular bunch spacing with $M$ bunches, $\tau$, is obtained by

$$
\begin{array}{r}
\tau_{\mu}^{-1}=\frac{e I_{0} \alpha}{2 E_{0} T_{0} \omega_{s}} \sum_{p=1}^{\infty}\left[\omega_{p}^{(\mu+)} \rho\left(\omega_{p}^{(\mu+)}\right) \operatorname{Re}\left\{Z\left(\omega_{p}^{(\mu+)}\right)\right\}-\omega_{p}^{(\mu+)} \rho\left(\omega_{p}^{(\mu+)}\right) \operatorname{Re}\left\{Z\left(\omega_{p}^{(\mu+)}\right)\right\}\right] \\
\omega_{p}^{(\mu+)}=\{(p-1) M+\mu\} \omega_{0}+\omega_{s}, \omega_{p}^{(\mu-)}=(p M-\mu) \omega_{0}+\omega_{s}, \\
\rho(\omega)=e^{-\omega^{2}\left(\sigma_{z} / c\right)^{2} / 2}, Z(\omega)=R_{s} N /\left\{1+i Q\left(\omega_{R} / \omega-\omega / \omega_{R}\right)\right\},
\end{array}
$$

where $\mu$ is the mode number, $I_{0}$ is the circulating current, $\alpha$ is the momentum compaction factor, $E_{0}$ is the beam energy, $T_{0}$ is the revolution period, $\omega_{\mathrm{s}}$ is the synchrotron frequency and $N$ is the number of BPMs. After evaluation of the mode number $\left(\mu^{\prime}\right)$, which gives the maximum 
growth rate $\left(\tau_{\mu}\right)$ for arbitrary $I_{0}$, the current limit, $I_{\max }$, can be obtained by

$$
I_{\max }=I_{0}\left(\tau_{\text {rad }} / \tau_{\mu}\right),
$$

where $\tau_{\text {rad }}$ is the radiation damping time $(20 \mathrm{~ms}$ for KEKB).

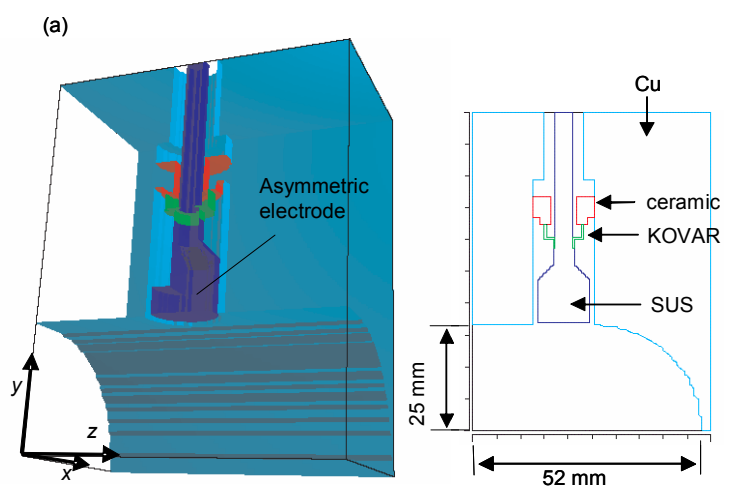

(b)

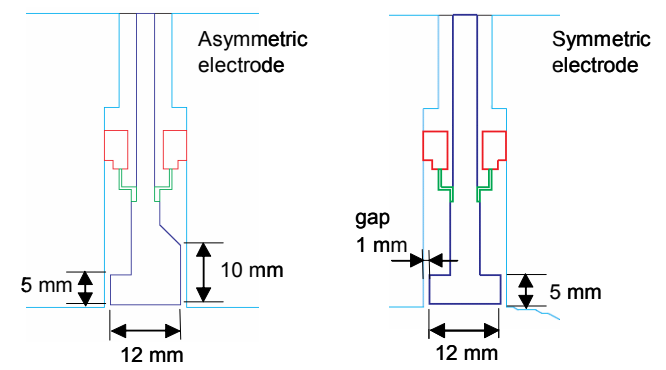

Figure 1: BPM for KEKB HER. (a) 3D cut model of asymmetric electrode and its cross-section ( $x-y$ plane). (b) Cross-section of the asymmetric and symmetric electrode (y-z plane).
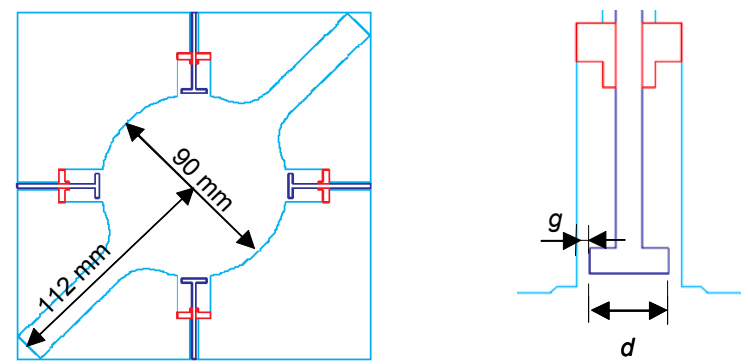

Figure 2: Cross-section of a beam duct with antechambers with BPMs for SuperKEKB modelled by MAFIA.

\section{Modeling}

An asymmetric button electrode is adopted for the BPM of KEKB HER, which can extract the electromagnetic energy of the TE110 mode into the coaxial cable as the propagation TEM mode [2]. The MAFIA model of the asymmetric BPM is shown in Fig. 1. The diameter of the disk is $12 \mathrm{~mm}$ and the gap size between the disk and wall is $1 \mathrm{~mm}$. The cross-section of the beam duct is the racetrack shape. We also calculated the longitudinal impedance of BPM with a symmetric button electrode for

05 Beam Dynamics and Electromagnetic Fields comparison. The cross-section of the symmetric button electrode is also presented in Fig.1. For the case of KEKB HER, only one quarter of the structure was calculated considering the symmetry. On the other hand, for Super $\mathrm{KEKB}$, the whole structure was simulated because the beam duct has two antechambers [3] and the coaxial line must be perpendicular to the boundary plane. The crosssection of the simulation model for the Super KEKB is shown in Fig.2. We used the symmetric electrode with diameter $(d)$ of $6 \mathrm{~mm}$ and $12 \mathrm{~mm}$, and the gap between the electrode and the wall $(g)$ is $1 \mathrm{~mm}$ and $2 \mathrm{~mm}$.

The wake potential was calculated up to $s=5 \mathrm{~m}$, where $s$ is the bunch coordinate. We used a Gaussian bunch with the bunch length $\sigma_{\mathrm{z}}$ of $6 \mathrm{~mm}$ and the charge $Q$ of $1 \mathrm{C}$. In the T3 solver, all metallic parts were considered to be perfect electric conductors. On the other hand, in the Eigenmode solver, the conductivity of the electrode, chamber and KOVAR part was set to $1.4 \times 10^{6} \Omega^{-1} \mathrm{~m}^{-1}$ (SUS), $5.6 \times 10^{7} \quad \Omega^{-1} \mathrm{~m}^{-1} \quad(\mathrm{Cu})$ and $2.0 \times 10^{6} \quad \Omega^{-1} \mathrm{~m}^{-1}$, respectively.

\section{RESULTS AND DISCUSSION}

\section{KEKB HER}

In Fig. 3 and 4, we show the longitudinal wake potential as a function of the beam coordinate $s$ and the corresponding longitudinal impedance spectrum. The longitudinal impedance has several resonances, but we focused on only a main resonance at around $7 \mathrm{GHz}$, and estimated the $Q_{\mathrm{T} 3}, R_{\mathrm{S} \_\mathrm{T} 3}$ and $f_{\mathrm{R}}\left(=\omega_{\mathrm{R}} / 2 \pi\right)$.

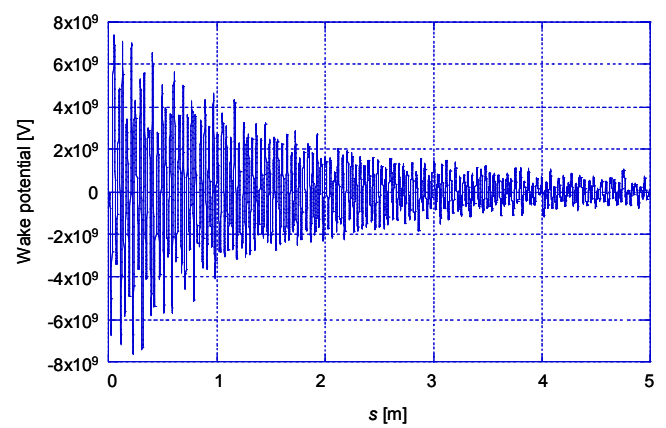

Figure 3: Longitudinal wake potential of asymmetric BPM for KEKB HER.

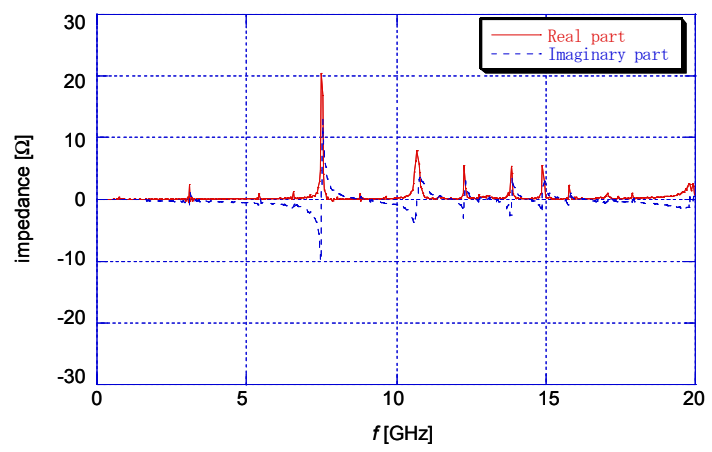

Figure 4: Longitudinal impedance spectrum of asymmetric BPM for KEKB HER. The solid and dotted lines are the real and imaginary parts, respectively.

D04 Instabilities - Processes, Impedances, Countermeasures 
Table 1: Longitudinal impedance of the BPMs for KEKB HER and the maximum beam current limited by the longitudinal MBI caused by the BPMs. $\left(E_{0}=8.0 \mathrm{GeV}, \alpha=3.4 \times 10^{-4}, T_{0}=1.0 \times 10^{-5} \mathrm{~s}, \omega_{\mathrm{s}} / 2 \pi=2 \mathrm{kHz}, N=443\right)$

\begin{tabular}{c|c|c|c|c}
\hline Electrode type & $\boldsymbol{f}_{\mathbf{r}}[\mathbf{G H z}]$ & $\boldsymbol{Q}_{\text {total }}$ & $\boldsymbol{R}_{\mathbf{s}_{\text {total }}}[\boldsymbol{\Omega}]$ & $\begin{array}{c}\boldsymbol{I}_{\mathbf{m a x}}[\mathbf{A}] \\
\left(\boldsymbol{\sigma}_{\mathbf{z}}=\mathbf{6} \mathbf{~ m m}, \text { by 4-buckets spacing }\right)\end{array}$ \\
\hline Asymmetric & 7.57 & 91.31 & 17.42 & 2.61 \\
Symmetric & 7.56 & 84.46 & 20.57 & 2.45 \\
\hline
\end{tabular}

Table 2: Longitudinal impedance of BPMs for the Super KEKB and the maximum beam current limited by the longitudinal MBI caused by the BPMs. $\left(E_{0}=3.5 \mathrm{GeV}, \alpha=3.4 \times 10^{-4}, T_{0}=1.0 \times 10^{-5} \mathrm{~s}, \omega_{\mathrm{s}} / 2 \pi=2 \mathrm{kHz}, N=443\right)$

\begin{tabular}{c|c|c|c|c|c}
\hline $\boldsymbol{d}[\mathbf{m m}]$ & $\boldsymbol{g}[\mathbf{m m}]$ & $\boldsymbol{f}_{\mathbf{r}}[\mathbf{G H z}]$ & $\boldsymbol{Q}_{\text {total }}$ & $\boldsymbol{R}_{\mathbf{s} \_ \text {total }}[\boldsymbol{\Omega}]$ & $\begin{array}{c}\boldsymbol{I}_{\mathbf{m a x}}\left(\boldsymbol{\sigma}_{\mathbf{z}}=\mathbf{3} \mathbf{~ m m}\right) \\
\mathbf{F u l l} / \mathbf{b y}-\mathbf{4}\end{array}$ \\
\hline 6 & 1 & 12.9 & 23.28 & 2.0 & $7.28 \mathrm{~A} />10.1 \mathrm{kA}$ \\
6 & 2 & 13.0 & 9.18 & 1.53 & $639 \mathrm{~A} />24.4 \mathrm{kA}$ \\
12 & 1 & 7.35 & 48.31 & 10.18 & $0.52 \mathrm{~A} / 2.39 \mathrm{~A}$ \\
12 & 2 & 7.50 & 36.49 & 8.60 & $0.69 \mathrm{~A} / 8.90 \mathrm{~A}$ \\
\hline
\end{tabular}

The Eigenmode solver computed twenty eigenmodes in the model, and from them we picked up TE110 mode which was trapped at the button electrode. The magnetic filed pattern of the TE110 mode is shown in Fig. 5. MAFIA can calculate the field energy $W[\mathrm{Js}]$ and the total dissipated power $P[\mathrm{~J}]$ of this mode, then $Q_{\mathrm{E}}$ was obtained by

$$
Q_{\mathrm{E}}=2 \pi f_{\mathrm{r}} W / P .
$$

The results are listed in Table 1 . When the electrode had the asymmetric shape, the Q-value and the shunt impedance were estimated at 91 and $17 \mathrm{ohm}$ (at 7.6 GHz), respectively. In this case, the current limit was $2.6 \mathrm{~A}$ for four RF-buckets spacing operation, which is a typical fill pattern of KEKB. For comparison, the current limit for symmetric electrode was also estimated, and it was $2.45 \mathrm{~A}$ for four buckets spacing operation. That is slightly lower than that of the asymmetric electrode, but the difference between them is small, $150 \mathrm{~mA}$. This result indicates that the asymmetric electrode is not indispensable. If the current limit is high enough, the symmetric electrode seems better for its simple structure and low manufacturing cost.

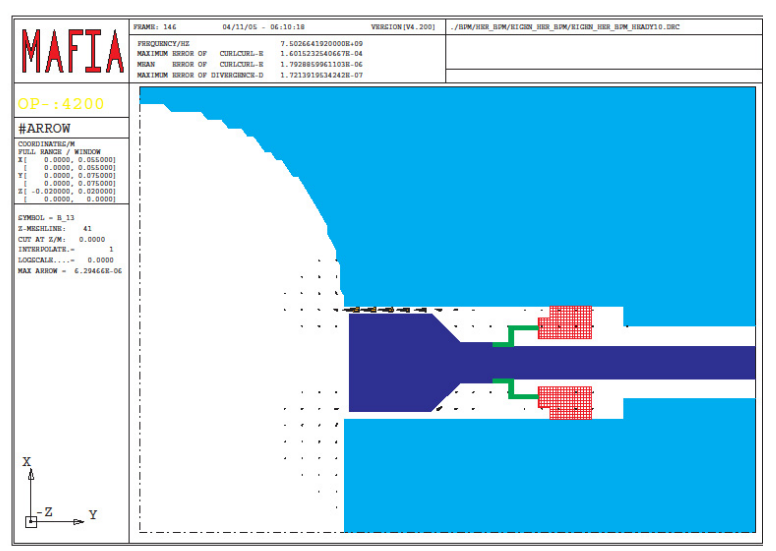

Figure 5: $y$ component of magnetic field of the TE110 mode calculated by the Eigenmode solver.

\section{Super KEKB}

The results for the Super KEKB are summarized in Table 2. When the diameter of the electrode was $12 \mathrm{~mm}$, the current limit was much smaller than the design current (LER:9.4 A, HER:4.1 A). On the other hand, the current limits for diameter of $6 \mathrm{~mm}$ were much larger than those of $12 \mathrm{~mm}$, and it was found that making button size smaller had a good effect on reduction of the longitudinal impedance. A larger gap size can reduces the Q-value at the resonance frequency. However, the smaller gap is better from the viewpoint of the sensitivity calculation of BPM by the boundary element method. If the diameter is $6 \mathrm{~mm}$ and gap is $1 \mathrm{~mm}$, the current limit was estimated to be over $10 \mathrm{kA}$ for four buckets spacing operation.

Based on the calculation, the new BPM electrode was designed. The diameter of the electrode was $6 \mathrm{~mm}$ and the gap size was $1 \mathrm{~mm}$. During the summer shutdown in 2007, the test chambers with two antechambers equipped with the new BPMs will be installed in a wiggler section. and this new BPM was used in this test chambers. The capability of this new BPM will be tested in the following autumn run.

\section{REFERENCES}

[1] C. - K. Ng, T. Weland, D. Martin, S. Smith and N. Kurita, "Simulation of PEP-II Beam Position Monitors", SLAC-PUB-95-6899, May 1995.

[2] T. Obina, T. Shintake, Y. H. Chin and N. Akasaka, "Damped Button Electrode for B-Factory BPM System", PAC95, Dallas, Texas, May 1-5, 2005, p. 2613, http://www.jacow.org.

[3] Y. Suetsugu, K. Kanazawa, K. Shibata, N. Ohuchi, H. Hisamatsu, M. Shirai, "R\&D Status of Vacuum Components for the Upgrade of KEKB" PAC05 Knoxville, Tennessee, 2005 May 16-20, p. 3256, http://www.jacow.org. 